\title{
Proven Intra and Interobserver Reliability in the Echographic Assess- ments of Body Fat Changes Related to HIV Associated Adipose Redistri- bution Syndrome (HARS)
}

\author{
Rosario Gulizia ${ }^{*},{ }^{1}$, Alessia Uglietti ${ }^{2}$, Antonella Grisolia ${ }^{1}$, Cristina Gervasoni ${ }^{3}$, Massimo Galli ${ }^{3}$ and \\ Carlo Filice ${ }^{1}$
}

\begin{abstract}
${ }^{I}$ Division of Infectious and Tropical Diseases, Foundation IRCCS S Matteo, University of Pavia, Italy; ${ }^{2}$ Division of Infectious Diseases, Foundation IRCCS S Matteo, University of Pavia, Italy; ${ }^{3}$ Infectious and Tropical Disease Institute, IRCCS L. Sacco, University of Milano, Italy
\end{abstract}

\begin{abstract}
Objective: To prove intra- and inter-observer's reliability of ultrasound (US) in the assessment of lipoatrophic findings related to the HIV associated Adipose Redistribution Syndrome (HARS).

Patients and Methods: In two separated sessions, 2 consecutive measurements of subcutaneous fat thickness (SFT) were performed by each observer at the deepest point of Bichat pad, the dorsal face of arm and the mid thigh for the assessment of facial, brachial and crural lipoatrophy, respectively. We enrolled 20 HIV patients, rotating an experienced and untrained sonologist. The assessments were performed avoiding any stand off pads in the skin and excluding artefacts due to the too abundant quantity of gel to obtaining, with minimal transducer pressure, the best resolution of the reference points.

Results: Means of facial, brachial and crural SFT showed no significant differences between the workers. Coefficients of variability $(\mathrm{SD} /$ mean $\mathrm{x} 100)$ were similar for facial (ranges: $4.7-5.2 \%$ vs $4.9-5.6 \%$, respectively), brachial (ranges: 5.8 $8.4 \%$ vs 9.7-11.2\%) and crural SFTs (ranges: $5.9-6 \%$ vs 6.2-8.7\%). There was greater consistency in the measurements performed by the experienced $v s$ the untrained worker. Inter-observer agreement, assessed through kappa statistic (k) analysis, confirmed increased measurement's agreement in the facial (k ranged from 0.40 to 0.60 ), brachial (k: 0.23-0.63) and crural SFT assessments (k: 0.58-0.70) from the $1^{\text {st }}$ to $2^{\text {nd }}$ session.

Conclusions: US shows low intra observer variability and good inter observer reliability in the assessment of body fat changes related to the HARS. The different degree of consistency by the workers and the improvement of interobserver agreement, suggest to stating a well defined period of training to obtain better US reliability.
\end{abstract}

Keywords: Sonography, Lipodystrophy, intra and inter-observer reliability, antiretrovirals, body fat changes.

We read the interesting report by Padilla et al. [1] about the use of ultrasound (US) in assessing of body fat changes (BFCs) related to HIV associated Adipose Redistribution Syndrome (HARS) [2, 3].

The authors confirm the good accuracy of US [4-10], further validating our opinion that the routine US assessment of related to antiretroviral (ARV) therapy should be part of the standardized procedures in the care of HIV patients. However, some open questions stay on:

1) Computed tomography (CT) is the gold standard tool for the BFCs assessments based on its low variability and high accuracy $[11,12]$. Anyhow, recent reports [4-10] have demonstrated the utility of alternative methods such as US, that are easier to perform and more readily available both of which are valuable assets when close follow up is required.

During the 12th Conference on Retroviruses and Opportunistic Infections, i.e., Moyle et al. reported the ability of US to document the recovery of malar

\footnotetext{
*Address correspondence to this author at the Division of Infectious and Tropical Diseases, Foundation IRCCS S. Matteo University of Pavia School of Medicine, Viale Taramelli 5, 27100 Pavia, Italy; Tel: +39 382 502887;

E-mail: rosario.gulizia@libero.it
}

subcutaneous fat thickness (SFT) at deepest point of Bichat pad in patients who had been switched to less invaliding new generation of ARVs, using 6 months re-assessments which are not readily feasible with CT.

2) Interestingly, in the few studies that have compared US with CT in measuring SFT $[1,13]$ the methods proved comparable; however the authors obtained controversial results when assessing malar SFT at the deepest point of Bichat pad [1]. We feel that the question of measurement's technique is crucial to explain these results. Based on our measurement's technique, i.e., our data suggest high comparability between the CT and US measurements of SFT at reference points (RPs) representative of facial, brachial and crural lipoatrophy (LA) (Fig. 1a-c) [14].

3) Proven, reliable and well standardized RPs have been validated for brachial and crural LA [9], as well as confirmed also by Padilla et al. [1]. The RP for facial LA is more controversial.

In previous reports [8] we observed that US scannings of deepest point of Bichat's pad provide reliable evidence for diagnosing of facial SAT loss (Fig. 1a). In our investigation we are observing good accuracy of 
(A)

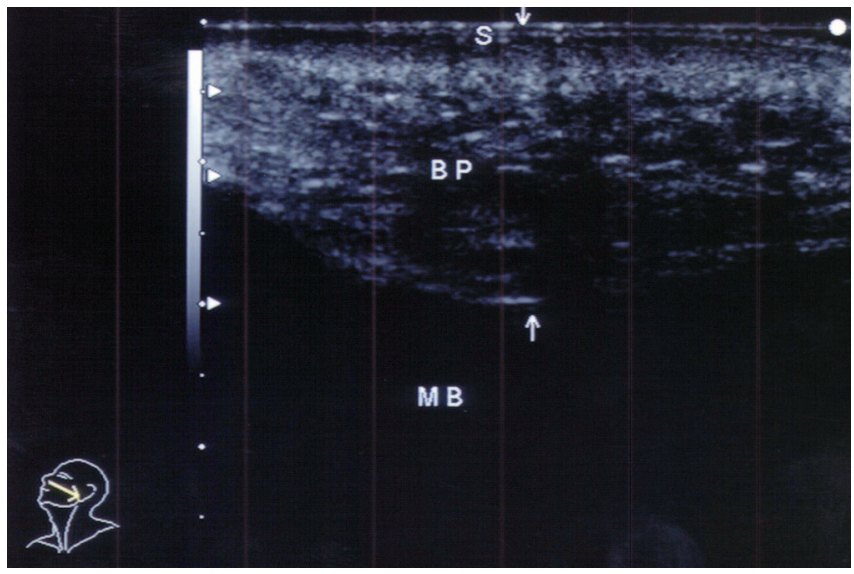

(B)

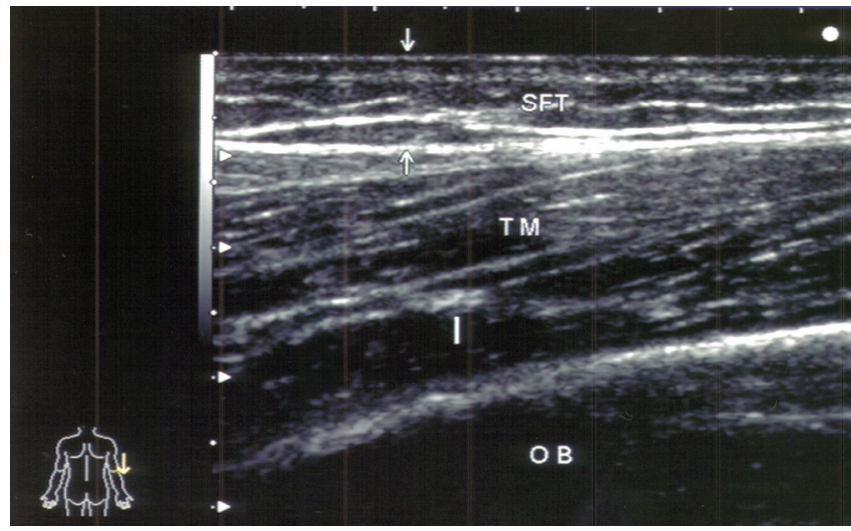

(C)

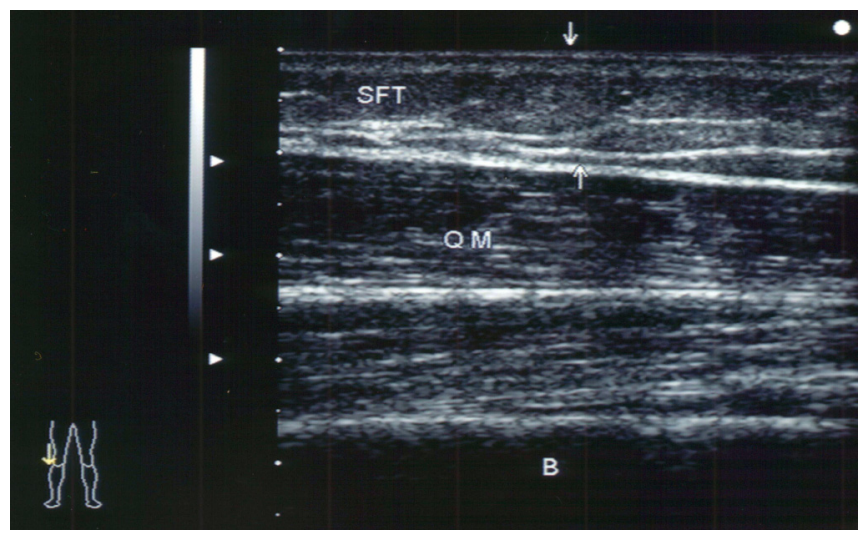

Fig. (1). Sonographic reference points for the assessment of subcutaneous fat thickness related to the body fat changes described in the HARS. US scan with $7.5-13 \mathrm{MHz}$ linear transducers is performed with no pressure on the underlying skin. (A) Facial LA: SFT is measured with a left nasogenian transversal scan from the malar bone (MB) to the hyperechoic line corresponding to corneous layer of epidermidis (S) on deepest point of Bichat pad (BP). (B) Brachial LA: SFT is measured with a long scans 10 centimetres above the right elbow from superficial fascia of triceps muscle ( $T$ $\mathrm{M})$ upper omeral bone (OB) to the hyperechoic line corresponding to corneous layer of epidermidis (S). (C) Crural LA: SFT is measured with long scans 15 centimetres above the rotula, from superficial fascia of quadriceps muscle (QM) upper the femoral bone (B) to the hyperechoic line corresponding to corneous layer of epidermidis (S).
(A)

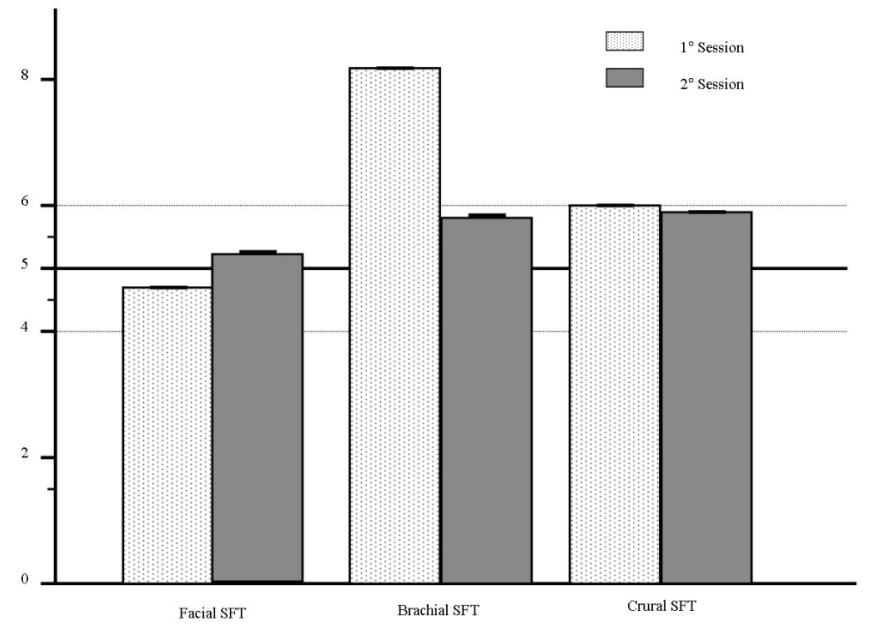

(B)

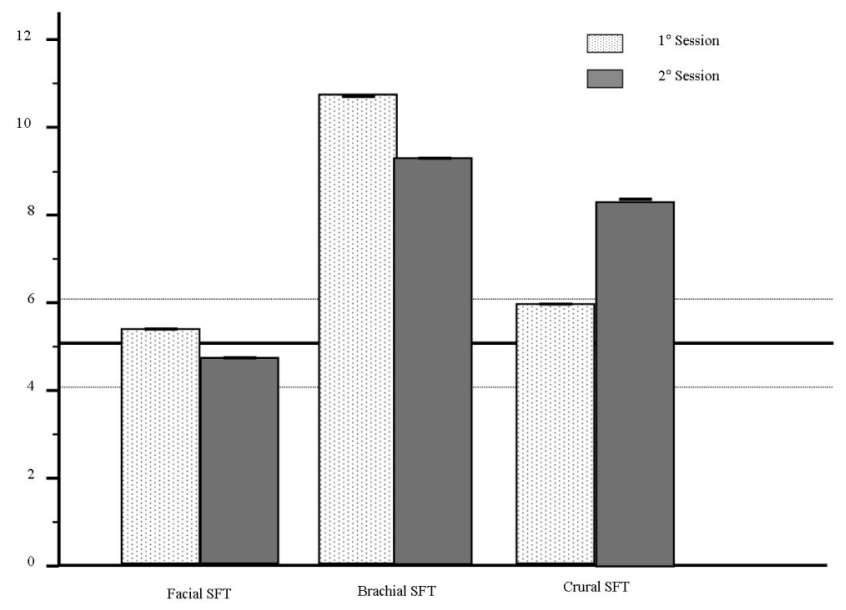

Fig. (2). Intra observer variability of Experienced and Untrained Sonologist in the sonographic measurements of subcutaneous fat thickness.

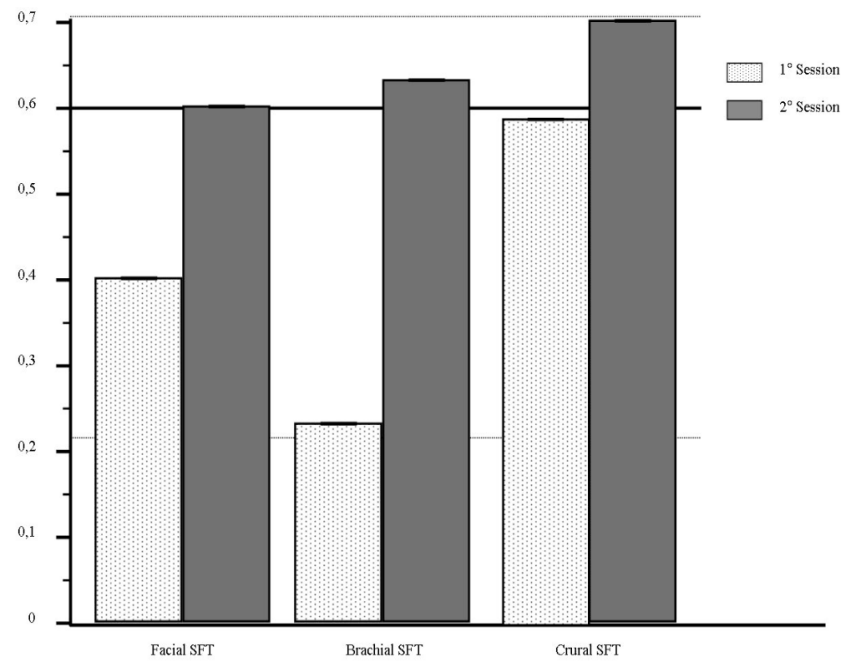

Fig. (3). Inter rate agreement (kappa) between the Sonologists in the single sessions. The agreement was quantified by the kappa $(k)$ statistic: $\mathrm{k}$ is 1 when there is perfect agreement between the classification system; 0 when there is no agreement better than chance; the strength of agreement is poor when $\mathrm{k}<0.20$, fair when $\mathrm{k}<0.40$, moderate when $\mathrm{k}<0.60$, good with $\mathrm{k}<0.80$, excellent with $\mathrm{k}>0.80$. 
this facial SFT measurement's technique and it seem to correctly diagnose high percentages of facial LA compared to the clinical assessments. The high level of agreement with the CT assessments suggests that it is simple and more reliable than more peripheral RPs $[6,8]$.

4) Therefore, although the authors [1], that assessed the same RP, shows disappointing results, we feel that a not optimized measurement's technique could have influenced their results. This opinion is strengthened by the high intra- and inter-observer variability reported by Padilla et al. [1] ( $\sim 31 \%$ and 32\%, respectively based on 6 assessments by 3 radiologists for only 2 patients) as compared to that observed in other studies (2.6-3.5\%) [9]. We investigated intra and inter-observer's variability of facial, brachial and crural SFT assessments made by an experienced and an untrained sonologist. Each sonologist performed 3 consecutive measurements of 20 lipoatrophic patients during two separate sessions, using an EUB 8500 (HITACHI Medical System). Coefficients of variability $(\mathrm{SD} /$ mean $\mathrm{x} 100)$ were similar for the facial (ranges: $4.7-5.2 \%$ vs $4.9-5.6 \%$, respectively), brachial (range $5.8-8.4 \%$ vs $9.7-11.2 \%$ ) and crural SFTs (ranges $5.9-6 \%$ vs $6.2-8.7 \%$ ). There was greater consistency in the measurements performed by the experienced $v s$ the untrained worker suggesting the need for standardized training to achieve the better reliability (Fig. 2a,b).

Inter-observer agreement as assessed by means of kappa statistic (k) analysis confirmed increased measurement agreement in the assessments of facial ( $\mathrm{k}$ ranged from 0.40 to 0.60$)$, brachial (k: $0.23-0.63$ ) and crural RP (k: 0.58-0.70) from the $1^{\text {st }}$ to the $2^{\text {nd }}$ session with definitive good agreement (Fig. 3). We are currently enlarging our experience in a multicentric study with similar encouraging reports on the efficacy of training performed by the same experienced teacher.

In conclusion, if several reports [4-10] show that US can be used to accurately identify HARS-related BFCs, the use of a well-defined and standardized measurement technique is required in order to optimize the reliability and utility of USmediated BFC assessment, beginning with the careful identification and measurement of RPs, avoiding any bias such as the pressure offered by stand off pads in the skin, and taking care in excluding artefacts due to the too abundant quantity of gel in order to obtain, with minimal transducer pressure, the best resolution of RPs such as indicated in Fig. (1).

\section{REFERENCES}

[1] Padilla S, Gallego JA, Masia M, Ardoy F, Hernandez I, Gutierrez F. Ultrasonography and anthropometry for measuring regional body fat in HIV-infected patients. Curr HIV Res 2007; 5(5): 459 66.

[2] Lichtenstein K, Balasubramanyam A, Sekhar R, Freedland E. HIVassociated adipose redistribution syndrome (HARS): etiology and pathophysiological mechanisms. AIDS Res Ther 2007; 4: 14

[3] Lichtenstein K, Balasubramanyam A, Sekhar R, Freedland E. HIVassociated adipose redistribution syndrome (HARS): definition, epidemiology and clinical impact. AIDS Res Ther 2007; 4: 16.

[4] Asensi V, Martin-Roces E, Carton JA, et al. Perirenal fat diameter measured by echography could be an early predictor of lipodystrophy in HIV type 1-infected patients receiving highly active antiretroviral therapy. Clin Infect Dis 2004; 39(2): 240-7.

[5] Asensi V, Martin-Roces E, Collazos J, et al. Association between physical and echographic fat thickness assessments and a lipodystrophy grading scale in lipodystrophic HIV patients: practical implications. AIDS Res Hum Retroviruses 2006; 22(9): 830-6.

[6] Carey D, Wand H, Martin A, et al. Evaluation of ultrasound for assessing facial lipoatrophy in a randomized, placebo-controlled trial. AIDS 2005; 19(12):1325-7.

[7] Gulizia R, Brunetti E, Gervasoni C, Galli M, Filice C. Sonographic assessment of lipodystrophy in HIV-infected patients: some open questions. Clin Infect Dis 2005; 40(2): 323-4.

[8] Gulizia R, Vercelli A, Gervasoni C, et al. Controversy concerning role of ultrasonographic lipoatrophy assessments in HIV patients. AIDS 2006; 20(5): 789-90.

[9] Martinez E, Bianchi L, Garcia-Viejo MA, Bru C, Gatell JM. Sonographic assessment of regional fat in HIV-1-infected people. Lancet $2000 ; 356(9239)$ : 1412-3.

[10] Martinez E, Milinkovic A, Bianchi L, Gatell JM. Considerations about the value of sonography for the measurement of regional body fat. AIDS 2006; 20(3): 465-6.

[11] Padilla S, Gallego JA, Masia M, Gutierrez F. Single-slice computed tomography and anthropometric skinfold analysis for evaluation of facial lipoatrophy in HIV-infected patients. Clin Infect Dis 2004; 39(12): 1848-51.

[12] Padilla S, Gallego JA, Masia M, Gutierrez F. Techniques for measuring regional body fat: computed tomography may be suitable for evaluation of facial lipoatrophy. AIDS 2006; 20(17): 2237.

[13] Milinkovic A, Martinez E. Current perspectives on HIV-associated lipodystrophy syndrome. J Antimicrob Chemother 2005; 56(1): 69.

[14] Gulizia R, Vercelli A, Gervasoni C, et al. Comparability of echographic and tomographic assessments of body fat changes related to the HIV associated Adipose Redistribution Syndrome (HARS) in antiretroviral treated patients. Ultrasound Med Biol 2008; Feb 26 [Epub ahead of print]. 